\title{
Los académicos del Instituto de Ingeniería y Tecnología de la UACJ y el Sistema Nacional de Investigadores
}

\author{
The academics of the Institute of Engineering and Technology \\ of the UACJ and the National System of Researchers
}

\author{
H. M. Camarillo-Hinojoza ${ }^{1}$ \\ 1 Universidad Autónoma de Ciudad Juárez
}

\section{RESUMEN}

El objetivo del presente artículo es presentar los hallazgos de investigación sobre algunas experiencias y problemáticas de los profesores-investigadores del Instituto de Ingeniería y Tecnología (IIT) de la Universidad Autónoma de Ciudad Juárez (UACJ), al ser evaluada su productividad por parte del Sistema Nacional de Investigadores (SNI). El artículo se deriva de una investigación más amplia consistente en un comparativo de las experiencias y problemáticas de los académicos de distintas áreas de conocimiento de dos universidades públicas al ser evaluado su trabajo académico por parte del SNI y otros dos programas de evaluación académica en México. Para la recolección y análisis de los datos se utilizó una metodología cualitativa de corte interpretativo sustentado en la teoría fundamentada (grounded theory). Es un artículo original porque en la literatura especializada no existe otro que aborde el objetivo presentado. Además aporta a la comprensión de las dificultades particulares de los académicos en mención. En los resultados se propone el concepto de estancamiento de la distinción, que emergió a partir del proceso de análisis. Las limitaciones de este trabajo recaen en que los resultados y conclusiones no pueden generalizarse debido al empleo de una metodología cualitativa en el contexto de una organización académica.

PALABRAS CLAVE: Sistema Nacional de Investigadores; productividad; evaluación académica; áreas de ingeniería.

\begin{abstract}
The objective of this article is to present the research findings on some experiences and problems of the professor-researchers of the Institute of Engineering and Technology (IIT) of the Universidad Autónoma de Ciudad Juárez (UACJ), when their productivity is evaluated by the System National Investigators (SNI). The article is derived from a broader investigation consisting of a comparison of the experiences and problems of academics from different areas of knowledge from two public universities when their academic work was evaluated by the SNI and two other academic evaluation programs in Mexico. For the collection and analysis of the data, a qualitative methodology of an interpretive cut was used based on grounded theory. It is an original article because in the specialized literature there is no other that addresses the objective presented. It also contributes to the understanding of the particular difficulties of the academics in question. The results propose the concept of the stagnation of the distinction, which emerged from the analysis process. The limitations of this work lie in the fact that the results and conclusions cannot be generalized due to the use of a qualitative methodology in the context of an academic organization.
\end{abstract}

KEYWORDS: National Research System; productivity; academic evaluation; engineering areas.

Correspondencia:

DESTINATARIO: Hugo Manuel Camarillo Hinojoza INSTITUCIÓN: Universidad Autónoma de Ciudad Juárez DIRECCIÓN: Av. Universidad y Heroico Colegio Militar s/n, Zona Chamizal (ICSA, edif. J), Ciudad Juárez, Chihuahua, México, C. P. 32300 CORREO ELECTRÓNICO: hcamaril@uacj.mx
Fecha de recepción: 5 de septiembre de 2020. Fecha de aceptación: 13 de noviembre de 2020. Fecha de publicación: 19 de noviembre de 2020
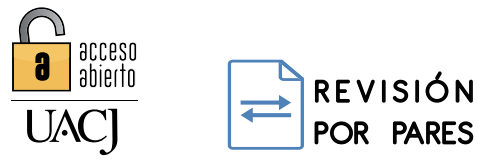

Licencia Creative Commons

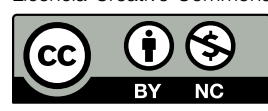




\section{INTRODUCCIÓN}

Conforme al artículo 13 del Reglamento del SNI, existen siete comisiones dictaminadoras correspondientes al mismo número de áreas del conocimiento reconocidas por este: I. Físico-Matemáticas y Ciencias de la Tierra; II. Biología y Química; III. Medicina y Ciencias de la Salud; IV. Humanidades y Ciencias de la Conducta; V. Ciencias Sociales; VI. Biotecnología y Ciencias Agropecuarias; y VII. Ingenierías [1], así como una comisión trasversal que tiene por objeto evaluar el impacto del trabajo de investigación e innovación de temas comunes a todas las áreas y cuya descripción se encuentra en el artículo 14 de la misma reglamentación.

A su vez, cada área tiene determinados criterios de evaluación en los que se especifican los productos académicos y de investigación que se consideran para asignar el tipo de nombramiento otorgado y el nivel que corresponda [2]. Los criterios sirven de guía para la revisión de los expedientes que se presentan en cada convocatoria. Incluso, algunas de las áreas prevén criterios más rígidos que otras, ya que dependiendo de la comisión dictaminadora y el área de conocimiento de que se trate, interesa más - por ejemplo-valorar publicaciones en revistas con alto Factor de Impacto (FI) indexadas en el Journal Citation Reports (JCR), SCimago, Scopus, el Sistema de Clasificación de Revistas Mexicanas de Ciencia y Tecnología (CRMCYT) del Consejo Nacional de Ciencia y Tecnología (Conacyt), que publicaciones en revistas reconocidas por otros índices internacionales o libros en editoriales de reconocido prestigio [2]. Por ejemplo, en las áreas I (Físico-Matemáticas y Ciencias de la Tierra) y VII (Ingenierías) se solicitan regularmente los parámetros más estrictos para evaluar los productos que en las demás, debido a que se ponderan los artículos científicos publicados con alto FI en JCR, SCimago y Scopus, entre otros. Es decir, artículos publicados en revistas de cuartiles Q1, Q2, Q3 o Q4, revistas del CRMCYT, libros o capítulos publicados en editoriales de prestigio y desde luego patentes y desarrollos tecnológicos o patentes reconocidos por la comisión transversal de tecnología [2], [3].

En este sentido, se advierte que la productividad es imprescindible para ingresar, permanece o ascender de nivel en el SNI, pero ante las diferencias entre las distintas áreas del conocimiento y sus investigadores debido a sus dinámicas académicas, formas de hacer investigación, aproximaciones epistemológicas y los criterios con los que se evalúa su trabajo académico, entre otros aspectos, resulta necesario y enriquecedor la comprensión de las problemáticas que viven los académicos de diferentes áreas del conocimiento durante el proceso de evaluación para ingresar y permanecer o escalar en el SNI. Bajo este contexto, el objetivo de este trabajo es presentar los hallazgos de investigación sobre las experiencias y problemáticas de los profesores-investigadores del IIT de la UACJ al ser evaluada su productividad por parte del SNI. En esto recae la pertinencia e importancia del presente artículo, ya que se ha identificado una ausencia de este tema en la literatura especializada publicada.

Haciendo un recuento del organismo evaluador que ejecuta la política científica y tecnológica en México, su creación está documentada oficialmente por medio de un decreto presidencial a finales de julio de 1984 [4], [5]. Surgió en medio de una crisis económica que afectó, entre otras cosas, los salarios de la profesión académica, por lo que fue diseñado para tratar de resolverla mediante la distribución de estímulos de manera diferenciada, según la productividad de los investigadores [6], [7]. Algunos años después de realizarse la primera evaluación para pertenecer a la élite de investigadores en México, se comenzaron a publicar artículos que hacían referencia a sus efectos perversos. Así le llamaron algunos autores porque se trataban de efectos no esperados que, desde sus perspectivas, hacían problemático el proceso de evaluación al grado de emerger posicionamientos críticos. Se trataban de los efectos causados entre los académicos evaluados.

Por ejemplo, De Ibarrola ${ }^{[8]}$ señaló dos tendencias de la evaluación del trabajo de los académicos: una que advirtió como descalificadora y otra como justificadora. Para la autora, la primera consistía en premiar y reconocer a unos cuantos académicos y descalificar a los que no reunían los requisitos para obtener un resultado favorable en la evaluación. La segunda tendencia estaba relacionada con las exiguas condiciones equitativas para que los académicos pudieran generar productos de calidad para ser valorados por el SNI. Esto por las diferencias institucionales de los investigadores, ya que es distinto hacer investigación desde una universidad pública o desde los centros de investigación por las diferencias en la dedicación a la docencia y a la investigación [9].

A su vez, Gil [10] expuso que si bien la evaluación del SNI propiciaba tensiones y desconciertos, el proceso de 
"diferenciación" entre los académicos, consistente en otorgar diferentes niveles como investigadores según su productividad, era una estrategia pertinente para la educación superior, ya que debido a su expansión durante la década previa al SNI había una falta de regulación en la contratación de los profesores, lo que implicaba insuficiente competitividad y solidez académica.

Otros trabajos también externaron severas críticas hacia los procesos de evaluación del SNI. Luego de las primeras convocatorias, Glazman [11] afirmó que las comisiones dictaminadoras se inclinaban por valorar los productos derivados de metodologías de investigación positivistas en menosprecio de productos bajo metodologías de corte cualitativo. Además advirtió sobre el sentir de los académicos que son rechazados durante el proceso evaluativo y en el que aparece "la vergüenza de la descalificación o de la subcalificación” [11, p. 5].

Díaz [12] también coincidió con la autora mencionada en el párrafo anterior. Aseguró que la evaluación realizada por los pares se caracterizaba por prejuicios y descalificación entre grupos, además de relaciones de poder en las que se identificaba la intención por colocar prospectos en las comisiones dictaminadoras por parte de las comunidades académicas. Asimismo destacó problemáticas como la angustia de los evaluados y la perspectiva de: "dime cuánto publicas y te diré quién eres" $[12$, p. 9$]$ por parte de los evaluadores.

Desde la perspectiva de De Vries [13], luego de algunos años de la creación del SNI era complejo determinar si derivado de sus directrices se había mejorado la investigación o la productividad de los académicos. En su artículo expuso que para el año 2000 no había suficiente claridad sobre sus efectos positivos o nocivos.

A principios de ese año, Grediaga [14] también escribió acerca de la poca reflexión sobre los efectos de las evaluaciones del SNI en los académicos. Por su parte, Salord [15] enfatizó que debido al SNI y a otras políticas se hizo visible la importancia de la meritocracia en la profesión académica y en el desarrollo de las trayectorias, aspecto que de alguna manera había advertido Díaz [12].

Otros autores refieren el desgaste emocional de los miembros del SNI [16]. Reportaron que debido a la sobrecarga laboral, cerca del $20 \%$ de los investigadores que formaron parte de la muestra del estudio sufría de algún desgaste de carácter emocional. También encon- traron entre los investigadores cierta rivalidad y competencia propiciada por la búsqueda de la membresía como investigador nacional.

Como se puede advertir en la revisión de la teoría publicada, la evaluación a cargo del SNI a través de su política y reglamentación, pero ejecutada por medio de las comisiones dictaminadoras, tuvo impacto en el aspecto personal y académico de los investigadores desde las primeras evaluaciones realizadas. Incluso, 26 años después de la creación del SNI, Didou y Gérard [7] señalaron otro tipo de efectos. Sostuvieron en su trabajo que los investigadores incipientes emulan a sus colegas con reconocimiento consolidado por parte del SNI, porque desean construir un perfil de investigador.

En esto coincidieron Rincones y Camarillo [17]. Identificaron un notable interés de los investigadores noveles por obtener el nombramiento que otorga el SNI en virtud de la mimetización con los investigadores con reconocimiento y prestigio académico que tuvieron como profesores durante su formación doctoral. Conforme a estos autores, la admiración los motiva a participar en las convocatorias del Sistema en búsqueda de alguna de las distinciones siguiendo los pasos de sus directores de tesis. A esto le denominan idolatría del nombramiento.

En este sentido, conforme a Cárdenas [18], pertenecer al Sistema tiene como significado para los investigadores una legitimidad como tales aunque les implique estrés y angustia, dado que les garantiza a mediano o largo plazo una satisfacción ya sea por el estímulo económico o por el otorgamiento de la distinción.

Otros artículos también documentan las problemáticas que se suscitan en la vida académica de los investigadores [19]. Por ejemplo, presentan como hallazgos que durante su búsqueda por mantener vigente el nombramiento otorgado por el SNI, algunos académicos experimentan una incertidumbre y zozobra mientras dura el proceso de evaluación al no saber cuál será el resultado que emitirán los dictaminadores. El autor también refiere otras problemáticas como la necesidad de aparecer como primer autor en los artículos publicados, la urgencia de publicar en revistas con alto FI y la lucha por reunir la productividad suficiente para estar en posibilidad de ingresar y permanecer en el SNI [19].

El trabajo de Rodríguez et al. [20] coincide respecto a las dificultades que viven los académicos al ser evalua- 
dos. Resaltan la presión que experimentan "los investigadores por publicar rápida y continuamente, a fin de mantener un estatus y permanecer en un grupo selecto dentro de la academia” [20, p. 189]. Además, argumentaron que para incrementar la productividad es necesario el apoyo de la Institución de Educación Superior (IES) a la que están adscritos.

Hasta esta parte del artículo, puede identificarse que es determinante el impacto del SNI en la profesión académica debido a los comportamientos que se suscitan y han sido documentados. De acuerdo con Gil y Contreras [5], el SNI propició en cierta manera la homologación de los comportamientos de los investigadores de las diferentes áreas de conocimiento en búsqueda de un perfil de investigador.

Izquierdo y Atristán [21] también publicaron un artículo sobre las problemática y tensiones que viven las investigadoras en particular para su permanencia y ascenso en el SNI. Las autoras documentaron las tensiones por mantener la distinción. Además sostienen que para escalar en el Sistema es pertinente poner atención a los procesos de publicación, ya que encontraron frustraciones entre algunas de sus informantes al ser rechazados sus artículos por los dictaminadores de revistas. Recomiendan que para reducir este problema es necesario elegir cuidadosa y estratégicamente las revistas indexadas donde se desea publicar y diseñar un plan de trabajo anual que incluya proyectos de investigación para poder publicar sus avances por etapas en búsqueda de productividad. También refieren la urgencia de dirigir tesis y publicar artículos con sus tutorados.

Finalmente, Camarillo [3] documentó algunas problemáticas en los investigadores de la Universidad Autónoma de Baja California (UABC) que los llevaron a buscar pertenecer a la élite de investigadores en México. Destaca el deseo de ser legitimados como investigadores, la necesidad de equilibrar las desventajas que unos tienen respecto a otros en la competencia académica y la exclusión en los grupos de investigación que propicia la autopropulsión para investigar, escribir y publicar.

A partir de lo expuesto en este apartado introductorio, se desprende que la evaluación del trabajo de los académicos que realiza el SNI desde 1984, ha producido diferentes efectos y comportamientos entre los investigadores, por lo que es pertinente conocer, comprender y analizar las experiencias, problemáticas e interpre- taciones de los profesores-investigadores cuyo trabajo académico se realiza en el entorno de las áreas de ingeniería de las IES. En esto resalta la originalidad, pertinencia e importancia del presente artículo, ya que se focaliza en los académicos del IIT de la UACJ.

El artículo se divide en cuatro partes. La primera corresponde a esta introducción. Consiste en una breve revisión del estado del arte desde la creación del SNI hasta la actualidad respecto a lo que se ha publicado en relación a problemáticas o experiencias que experimentan los investigadores al ser evaluada su productividad por el SNI. Asimismo, se destaca lo inédito del artículo. En la segunda parte se hace referencia a la metodología utilizada, misma que fue de tipo cualitativa con enfoque interpretativo. Se describen aspectos relacionados con los informantes y el análisis de los datos, entre otros aspectos. En la tercera parte se presentan los hallazgos derivados del proceso de análisis. Además se destacan algunas de las voces de los informantes por la relevancia de sus testimonios y el significado de lo que expresan en su discurso. En este apartado se abordan temas como las elaboradas estrategias de publicación de los académicos de ingeniería, la discusión entre "publicar o perecer" e investigar para resolver problemas reales, la metamorfosis del publish or perish al direct thesis or perish, la rigurosidad de los criterios de evaluación de las áreas I y VII del SNI, la lucha y competencia por el financiamiento para desarrollar investigación ante los altos costos de los reactivos de laboratorio, entre otras experiencias. Además se presenta el concepto de estancamiento de la distinción, mismo que surgió a partir de las narrativas de los informantes, del proceso de categorización analítica y de las interpretaciones realizadas. En la cuarta y última parte se plantean algunas conclusiones, las limitaciones del estudio, así como las líneas que se pudieran seguir a partir de este trabajo.

\section{METODOLOGÍA}

Para realizar la investigación de la que deriva el presente artículo se utilizó una metodología cualitativa de corte interpretativo. Dicha investigación fue amplia y profunda dado que su objetivo consistió en comparar las problemáticas, perspectivas y experiencias de académicos de la UACJ y la Universidad Autónoma de Baja California (UABC), al ser evaluado su trabajo académico por parte del SNI, el Programa de Estímulos al Desempeño Docente (ESDEPED) y el Programa para el Desarrollo Profesional Docente de Tipo Superior 
(Prodep). Sin embargo, es pertinente precisar que, para los efectos del presente artículo, solo se hace referencia a los resultados obtenidos en uno de los entornos institucionales (la UACJ) y exclusivamente respecto al SNI en relación con actores en los que se focaliza este trabajo (los académicos del IIT). Esto conforme a lo expresado como objetivo del presente artículo y que se plantea y refiere en los apartados del resumen-abstract y la introducción.

Durante todo el proceso de la investigación original recurrimos a la grounded theory (teoría fundamentada) con base en las recomendaciones de Strauss y Corbin [22]. Esto nos permitió una aproximación epistemológica y un análisis de los datos emergentes utilizando diferentes marcos teóricos, por lo que los resultados se fueron construyendo a través de un proceso dialógico constante entre el dato y la teoría de corto, mediano y largo alcance como recomiendan los autores en cita.

Debido a que la investigación se situó en un enfoque interpretativo, se utilizó la entrevista en profundidad como técnica cualitativa para interactuar con 29 informantes con la idea de comprender sus voces y testimonios a través de los cuales le dan sentido y significado a sus experiencias [22] con las políticas y programas de evaluación académica.

Del total de entrevistados, 24 eran académicos y cinco funcionarios universitarios; 15 pertenecientes a la UABC y 14 a la UACJ. Fueron seleccionados con categoría como académicos y se ponderó diferente área del conocimiento, adscripción al interior de las IES, antigüedad, edad, género, experiencia, etc. Esto con la finalidad de que estuviera presente la heterogeneidad en los informantes. En cuanto a los funcionarios universitarios, se escogieron aquellos con actividades relacionadas con el SNI, el ESDEPED o el Prodep.

El uso de la entrevista en profundidad permitió que los informantes pudieran compartir múltiples aspectos, situaciones y experiencias relacionadas con su profesión académica sujeta a constantes evaluaciones.

Para el análisis de los datos se llevó a cabo un proceso de categorización abierta, axial y selectiva ${ }^{[22]}$ que comenzó desde la primera transcripción de las entrevistas. Las categorías medulares que emergieron a partir del análisis de los datos en relación al Sistema fueron: ingresar, permanecer, escalar, salir y reingresar al SNI. Sin em- bargo, dada la extensión de los resultados, solo hacemos referencia a aspectos de las primeras tres categorías.

Finalmente, cabe mencionar que cuando se cita el testimonio de algún informante se utilizan los denominativos de sujeto A, sujeto B y sujeto $C$, con la finalidad de resguardar su identidad.

\section{RESULTADOS Y DISCUSIÓN}

\subsection{Los académicos del área de ingeniería de la UACJ y el SNI}

En este apartado se presenta el análisis de algunas narrativas de profesores-investigadores que realzan actividades académicas en el contexto del Instituto de Ingeniería y Tecnología de la UACJ y cuya productividad es evaluada por el SNI.

El IIT está organizado en cuatro departamentos académicos: a) Física y Matemáticas; b) Ingeniería Civil y Ambiental; c) Ingeniería Eléctrica y Computación; y d) Industrial y Manufactura. Oferta 17 programas de pregrado, nueve maestrías y tres doctorados. Conforme a su página oficial [23] cuenta con un Centro de Investigación en Ciencia y Tecnología Aplicada (CICTA), un Centro de Información Geográfica (CIG), una instancia denominada: Unidad de Ingeniería del Conocimiento e Ingeniería del Software (UICIS) y siete laboratorios. Los profesores de tiempo completo (PTC) que desarrollan investigación en alguna de las diferentes áreas de generación y aplicación del conocimiento (LGAC) en el IIT pertenecen o han sido miembros del SNI en áreas como la I (Físico Matemáticas y Ciencias de la Tierra); IV (Humanidades y Ciencias de la Conducta); V (Ciencias Sociales) y VII (Ingenierías) [24]. Desde luego la extensa mayoría ha sido evaluada por la comisión dictaminadora del área VII, en menor grado por la I y solo algunos profesores-investigadores del IIT por las IV y V debido a que su perfil tiene rasgos interdisciplinarios entre la ingeniería y otras áreas del conocimiento [24].

Los resultados se presentan en ocho partes. Contribuyen al estado del conocimiento especializado dado que se identifican claramente las problemáticas y experiencias que viven los académicos de tiempo completo del IIT que además de realizar investigación, también dedican su tiempo a ejercer la docencia y otras actividades esenciales de la educación superior. Esto debido a que fueron contratados por una universidad considerada de docen- 
cia y no por centros de investigación, por lo que son académicos multifuncionales que apoyan a programas de pregrado y posgrado en distintas ramas de la ingeniería. En este sentido, a partir de sus testimonios sobre la evaluación de su trabajo académico por parte del SNI y del proceso de análisis, se presentan los siguientes resultados.

En algunas partes se citan textualmente los testimonios compartidos por los informantes debido a la relevancia y trascendencia de la narrativa. Además, para los efectos de este artículo se eligió utilizar principalmente la voz de uno de los informantes porque esta da cuenta significativamente y resalta en su discurso de manera clara y consiste las dificultades que afrontan los profesores-investigadores del IIT en su vida académica al ser evaluada su productividad por el SNI.

\subsection{La influencia para ingresar al SNI desde su formación doctoral}

Uno de los hallazgos en las narrativas de algunos entrevistados fue que la motivación para ingresar al SNI se configuró desde la formación doctoral, pues algunos de sus profesores contaban con el nombramiento como investigadores nacionales y con reconocimiento y prestigio académico. Una informante (sujeto A) compartió lo siguiente:

Eso fue desde el doctorado, desde el doctorado [...] porque empieza uno a oír: ¡Oye mira que fulanito tal es candidato, que es nivel uno! Y tú empiezas: ¿¿Pues qué es eso? ¿Qué es el Sistema Nacional de Investigadores? Y fue cuando empecé a involucrarme. Entonces cuando estaba en el doctorado pues ya sabía, ¿no?, que había el Sistema Nacional de Investigadores y que ahí estaba, que les daba [a los académicos] un apoyo y un reconocimiento para la investigación. [...]. Entonces dije: ¡Ah, voy a ingresar!

Esto se traduce en una motivación temprana en la búsqueda de la distinción como investigadora nacional quizá influenciada por sus profesores, pues la informante cursó el doctorado en una organización académica cuya planta docente tiene un alto número de SNI. Además, desde que la entrevistada era estudiante, estuvo rodeada de una dinámica investigadora competitiva en la institución donde se formó. Esto ocurre por la mimetización de los doctorantes con respecto a los académicos experimentados con capital universitario con quienes interactuaron durante su formación.
En la literatura especializada está documentado que la intención de pertenecer al Sistema regularmente se forja durante la formación doctoral. Pero lo relevante del hallazgo presentado en este artículo es que hay una ausencia de trabajos que refieran esta motivación de ingreso en los académicos de ingeniería. Algunos autores lo señalan en sus trabajos derivados de investigaciones [17], [18], [21]. Sobre todo destacan la influencia que el director de tesis ejerce en los doctorantes que buscan ingresar al SNI una vez que obtienen su grado. Se trata de investigadores noveles que tienen como objetivo comenzar una carrera dentro la élite científica.

\section{3. ¡Sin publicaciones no hay paraíso! Dime cuánto publicas y te diré qué nivel del SNI eres}

Pero no basta con el solo interés por ingresar al Sistema. Es obvio que existen algunos aspectos o requisitos que se deben cubrir para obtener una distinción del SNI, sobre todo un número consistente de publicaciones. La misma informante reveló que su productividad académica le permitió no solo ingresar al SNI, sino ubicarse en el nivel I sin pasar por el nivel de candidato, lo cual, desde su perspectiva, fue en reconocimiento a su trabajo:

Entrevistador: Cuando le dieron el nivel I en lugar de la candidatura, ¿cuál fue su impresión?

Sujeto A: La verdad es algo nuevo y satisfactorio porque dices bueno, me reconocen mi trabajo. Al final de cuentas es la única satisfacción que te queda ¿no?, bueno me reconocieron mi trabajo, yo trabajé, mi doctorado fue bastante duro, mi doctorado fue bastante bueno, entonces yo tuve suficientes artículos como para poder entrar al nivel uno.

Entrevistador: O sea, ¿durante el doctorado usted ya había publicado?

Sujeto A: Sí, bueno, en ciertas áreas, no sé si en tu doctorado te lo piden, pero por ejemplo en el doctorado [...] sí te exigen para poder darte el título de doctor, te piden dos artículos; entonces eso nos exigían a nosotros; dos artículos. Si, entonces ya con tres artículos puedes entrar en el área [tal al SNI]. Si tienes tres artículos y grado de doctor puedes entrar a candidato; si tienes cinco artículos o más de cinco artículos entonces ya te dan el nivel I. Entonces yo tenía más de cinco artículos de mi doctorado; fue de mi doctorado [...] y con eso me pusieron mi nivel uno. 
La informante fue enfática sobre la importancia de las publicaciones para ingresar al SNI. Incluso habla resaltando el aspecto cuantitativo, pero no el cualitativo. El número de publicaciones es un tema recurrente no solo de los profesores-investigadores de ingeniería sino de la generalidad de los académicos. Se trata de la relevancia de contar con el número de publicaciones suficientes para ingresar o mantener vigente su nombramiento dentro del SNI. En la profesión académica se ha difundido lo imprescindible que se ha vuelto la publicación de trabajos bajo la idea del publish or perish. Incluso, es una problemática que desde hace más de dos décadas ya había advertido Garfield [25] en 1996. Este autor se dio a la tarea de investigar quién había acuñado académicamente el término publish or perish. Encontró que había sido Logan Wilson, quien escribió por primera vez sobre "publicar o perecer" en su libro denominado El hombre académico. Estudio en la sociología de una profesión, publicado en 1942.

Casi en el mismo año que Garfield, Boyer [26] afirmó que la publicación y la investigación se convirtieron en actividades medulares de las IES y sus académicos debido al prestigio y reconocimiento que ambas pueden generar. Actualmente, es decir, 23 años después, la aseveración aún tiene vigencia en el contexto internacional y nacional de la educación superior, sus políticas y la evaluación académica. Ser académico se traduce en una dinámica consistente en la búsqueda por publicar, dado que esta actividad es el parámetro con que se mide la productividad académica por parte del SNI [19], [27].

La productividad es imprescindible ante los requerimientos de las políticas de evaluación académica. Incluso resalta la trascendencia de publicar en revistas con un alto FI para obtener un mejor resultado en los dictámenes de los evaluadores del SNI [27], el número de publicaciones logradas durante el periodo a evaluar y desde luego en qué espacios de publicación reconocidos y de prestigio se publica [3], [21].

\subsection{Algunas estrategias de publicación en el área de ingeniería de la UACJ}

Bajo este contexto, identificamos como hallazgo no documentado por la literatura especializada, que los académicos del área de ingeniería de la UACJ estructuran elaboradas estrategias de publicación. Estas son importantes para la profesión académica si es que se busca ingresar, permanecer o escalar en el Sistema. Si bien es cierto que se trata de una lógica académica propiciada por el utilitarismo académico [28] donde pareciera que quien más publica es quien tiene más estatus y prestigio [5], es pertinente resaltar y reconocer que si los académicos no se insertan en esta dinámica no podrían renovar sus nombramientos otorgados por el SNI.

Algunos de los informantes de otras áreas del conocimiento y de otros institutos, compartieron durante el trabajo de campo estrategias de publicación simuladas en las que se incorporan autores a las publicaciones sin haber mediado una participación real. Esta dinámica se presenta en la extensa mayoría de las IES de México y otras partes del mundo debido a la idea de "publicar o perecer", pero que autores como Day han criticado de forma severa:

A veces he visto 10 o más autores anotados en el encabezamiento de un artículo (que en ocasiones era solo una "nota"). Por ejemplo, un trabajo publicado por F. Bulos et al. (Phys. Rev. Letters 13:486, 1964) tenía 27 autores y solo 12 párrafos. Esos informes proceden con frecuencia de laboratorios tan pequeños que 10 personas no caben en ellos, ni mucho menos pueden hacer una contribución significativa al experimento. ¿A qué se debe la tendencia a enumerar una legión de autores? Puede haber varias razones, pero la principal, sin lugar a dudas, tiene que ver con el síndrome de publicar o perecer ${ }^{[29]}$.

En relación con el señalamiento de Day, encontramos un hallazgo que contraposiciona lo anterior, pues el sujeto A narró una estrategia seria y elaborada que no se sitúa en una simulación para aparecer en el paper, sino en una real estrategia de publicación y de permanencia en el SNI que no solo impacta favorablemente a nivel individual sino institucional. Se trata de la dinámica alrededor del posgrado que integra varios aspectos, lo que en conjunto permite ir configurando una garantía de infraestructura y laboratorios para realizar investigación y posteriormente publicaciones derivadas de esta actividad. Una de las informantes (sujeto A) compartió esta narrativa:

Sabíamos que queríamos hacer investigación, [...] te estoy hablando de [tal profesor], de [otro más] y una doctora que ya no está aquí y yo. Empezamos a trabajar en lo que es [...] investigación. ¿Qué necesitamos para hacer investigación? Obviamente ellos ya habían metido sus papeles al SNI y sabíamos que 
para mantenernos pues teníamos que publicar, y para publicar, pues necesitamos un montón de cosas ¿no? Necesitamos tener alumnos, necesitábamos laboratorios, necesitábamos financiamiento, necesitamos todo eso. Empezamos a trabajar en el programa de posgrado de la maestría, pues hay que hacer una maestría, necesitamos alumnos de la maestría.

$\mathrm{Al}$ analizar el testimonio anterior se percibe la pertenencia a un grupo en el sentido del tribalismo que refiere Maffesoli [30] y que busca consolidarse en materia de investigación en el campo de una rama de la ingeniería. Bajo este contexto, la entrevistada y sus colegas no solo intentan favorecerse mutuamente en el sentido académico y de la investigación con los proyectos y aspectos que menciona; se trata de confrontar colectivamente y de forma consistente los obstáculos y problemáticas que los rodean con el objetivo de mantenerse en el SNI a través de las publicaciones que se generan con el apoyo de lo siguiente: a) los laboratorios: ya que en el campo de la ingeniería son necesarios para realizar experimentos durante los proyectos de investigación; b) el financiamiento: es imprescindible, por ejemplo, para la compra de los reactivos que regularmente tienen un alto costo. Sin reactivos, el proyecto no sigue su curso; y c) la dirección de tesis: está previsto por los criterios de evaluación de todas las áreas reconocidas por el Sistema que para ascender de nivel es pertinente la dirección de tesis de estudiantes de pregrado o posgrado [2].

En este entorno, de los trabajos de investigación de los estudiantes conducidos por los directores de tesis pueden derivarse publicaciones entre ambos. Por tanto, es clara la estrategia del sujeto A y sus colegas para mantenerse vigentes en la profesión académica y permanecer en la élite de investigadores mediante la publicación de artículos, actividad que se ha configurado como difícil fundamentalmente para los investigadores pertenecientes a las áreas I y VII, debido a la rigurosidad de los criterios de evaluación de las comisiones dictaminadoras correspondientes.

\subsection{Publicar o perecer vs. investigar para resolver problemas reales}

Para otro de los informantes (sujeto B), la búsqueda de publicaciones conduce a plantear interrogantes como estas: ¿Se debe tener como objetivo solo publicar en virtud de la dinámica imperante en la profesión académica que conduce el SNI, o se debe hacer investigación para resolver problemas de la realidad? ¿Qué debe estar en primer término? El entrevistado advirtió que publicar no debe ser prioritario. Compartió que durante su posgrado se relacionó:

...con una investigación muy pragmática para resolver problemas, no para sacar papers. ¡Claro, de ahí salían! ¡Pero esa no era la preocupación! Ahorita es al contrario, o sea, ¿cuántos papers voy a sacar si hago esta investigación?

Después criticó a los científicos mexicanos. Afirmó que tienen abandonada la resolución de problemas:

Nosotros necesitamos gente práctica en este país. No gente que pase con mil papers y que le dieron la medalla Einstein y que no sirve para nada lo que hizo. Porque si nosotros vamos a evaluar el impacto de los productos científicos mexicanos; su impacto es casi cero. [...]. Nosotros [en México] producimos una cosa de ciencia que no es ciencia, que no tiene impacto, porque en este caso - me muestra en la pantalla de su computadora un artículo denominado: The scientific impact of nations de David King y me señala un listado- su impacto [se refiere a otras naciones] es tecnológico, su impacto es social, [...], ¡nosotros no producimos nada de eso! [...]. Porque si nos vamos a investigar el piojo del piojo, ¿si es nomás para eso?, joye, pues está fantástico!, pero si es para utilizarlo para controlar los piojos, bueno entonces ya traes algo práctico $\mathrm{Y}$ no es porque sea pragmático, porque en la realidad tenemos muchos problemas y no estamos resolviendo ni uno de ellos.

A partir del testimonio del sujeto B se advierte la crítica a la disociación del investigador con los problemas sociales por su necesidad lógica de conseguir que sean publicados sus artículos y reunir el número suficiente para estar en posibilidad de presentarlos para la convocatoria del SNI. El informante además agregó lo siguiente:

Si somos críticos y analizamos todas estas situaciones que se han dado [...], nosotros vamos a encontrar una historia interesante en el sentido del despego que hay del investigador de la realidad social, de la realidad del desarrollo industrial, de la realidad pues cotidiana. Y de cómo no tenemos visión para ver los problemas que se están ahorita creando, para avizorar que puede suceder. O sea, nosotros no somos en ese sentido previsores, no vemos el mañana. 
En otra parte de la entrevista criticó la inercia de publicar refritos, esto es, publicar un artículo que ya fue difundido en otra revista o con otros colegas y en el que la novedad solo consiste en que ha sido corregido o aumentado. El sujeto B señaló: "Yo creo que hay que tener una chispa creativa aunque nos equivoquemos. ¡Cámbiale el discurso! ¡Estamos cayendo en un disco rayado y publicamos lo mismo! Y si lo vemos, no sirve para nada".

Asimismo, el entrevistado también advierte que la búsqueda de productividad propicia que los investigadores se dejen llevar hacia líneas de investigación "del momento" por cuestiones coyunturales o de rentabilidad para publicar:

No hay agendas a veces. Y yo creo que es fundamental trabajar con agendas. Siempre va a haber imponderables, siempre va a haber la coyuntura de que: ¡Ay, se me antoja hacer esto!, pero yo creo que debe de haber una formalidad. Porque si nos mantenemos en una informalidad de ese limbo, que ocurre mucho en los centros de investigación y mucho en las universidades de México, entonces [...] iparece que hay productividad porque hay! ¡Pero no hay resultados que tengan impacto! Y menos de largo plazo. Por eso las agendas son importantes. Todo protocolizado y seguimientos estrictos.

Para el sujeto "B", publicar por publicar es una inercia generada por la ambición de obtener estímulos económicos. Argumenta que, como consecuencia, emergen investigadores impostores que persiguen publicar cuestiones intrascendentes para salir del paso y mostrar productividad. Propone sujetar a los investigadores a publicar un libro para cada evaluación para hacerlos reflexionar sobre lo que están publicando. El sujeto B habló con rigor:

Nos han metido a una inercia ambiciosa y de imposturas totalmente. Luego estamos inventando qué sacar, qué hacer, cómo salir del paso. Por ejemplo, yo digo que si nos obligaran a que en cada promoción publiquemos un libro individual, nos harían pensar en nuestra circunstancia y ser un poquito más útiles para la sociedad y para los estudiantes; pero no nos obligan. Menos del $10 \%$ de la gente que está en el SNI, tienen 10 libros individuales.

En resumen, se puede decir que el impacto del SNI en la vida académica de los PTC exhibe una pugna entre "in- vestigar para publicar vs. investigar para resolver problemas sociales", "publicar o dar respuestas". El testimonio de una funcionaria (sujeto C) coincide con el del sujeto $\mathrm{B}$, es decir, comenta que se debe ponderar la investigación para responder a las necesidades sociales. El sujeto C dijo: "Finalmente es para que haya una respuesta a la sociedad, o sea, no es nada más que se quede nada más en una torre de marfil y tener investigadores por tener investigadores, sino viene la otra parte: la transferencia, la utilización de ese conocimiento".

\subsection{La rigurosidad de los criterios de evaluación de las área I y VII del SNI}

El sujeto B propuso voltear la mirada a publicar libros, sin embargo, los criterios de evaluación de las áreas I y VII donde se concentran la mayor parte de los académicos del IIT que pertenecen al SNI, ponen en el centro de atención a los artículos publicados en revistas de alto FI. Incluso, se observa de manera determinante la rigurosidad de estos criterios si se comparan con los de otras comisiones dictaminadoras [2]. Por ejemplo, para el caso del área VII los productos que son aceptados por los evaluadores para poder ingresar a la élite científica son patentes o desarrollos tecnológicos que haya validado la comisión transversal, así como artículos del JCR que pertenezcan al cuartil Q1, Q2, Q3, Q4 o artículos del CRMCYT, así como también se ponderan los libros o capítulos de libros publicados en editoriales de reconocido prestigio [2]. Los parámetros de las revistas de los cuartiles en cita implican que los artículos ahí publicados deben ser de calidad debido a lo estricto de su proceso de dictaminación.

A su vez, en los criterios de evaluación del área I (Físico-Matemáticas y Ciencias de la Tierra) se identifican además parámetros rígidos como en el caso del área VII para evaluar los productos, ya que se ponderan los artículos científicos de Física y Ciencias de la Tierra publicados con FI 1 en JCR y para Matemáticas los de FI de 0.25 para ingresar al Sistema. Para ambas disciplinas también se consideran los artículos publicados en revistas de cuartiles Q1 y Q2 de SCimago y un número muy limitado de otras publicaciones con menor factor de impacto. Otros productos que son considerados son revistas mexicanas con editorial reconocida, libros o capítulos publicados en editoriales reconocidas y desde luego desarrollos tecnológicos o patentes reconocidos por la comisión transversal de tecnología [2]. 
Por supuesto que cuando se hace referencia a los altos parámetros de los criterios de evaluación de las áreas I y VII no significa que en las demás no haya rigurosidad. Incluso en otras los criterios también cuentan con un parámetro parecido. Sin embargo, conforme a los documentos que guían las evaluaciones de los productos disponibles en la página oficial del SNI, en otras áreas del conocimiento científico los criterios son más abiertos. Esto es comprensible debido a las diferentes dinámicas de cada área disciplinar, a sus formas de aproximación epistemológica y a sus propios ejercicios de evaluación. Se pueden consultar algunos estudios sobre el SNI, la productividad científica y los índices élite de las revistas científicas y tecnológicas con alto FI en las diferentes áreas del conocimiento [27], [31], [32], entre otros. Se trata de una tendencia. Incluso Cabrero ${ }^{[33]}$ señaló la predisposición del SNI a ponderar el impacto bibliométrico de las revistas donde publican los investigadores que son evaluados. Y precisamente, conforme a los criterios de evaluación de las áreas I y VII disponibles en la página web del SNI, el FI es uno de los aspectos medulares que se valoran en estas áreas del conocimiento en relación con la actividad de publicar.

\subsection{La lucha por el financiamiento y su impacto en la acción de publicar}

Retomando el tema del financiamiento que ya se había adelantado en el apartado 3.4, encontramos un hallazgo con la dualidad publicación-recurso. El financiamiento juega un papel trascendente para sostener la membresía como investigador nacional. El siguiente testimonio comprende la manera de cómo el recurso se entrelaza con las publicaciones, los proyectos de investigación, la infraestructura de laboratorio, los alumnos de posgrado y la urgencia latente de contar con posgrados reconocidos por el Programa Nacional de Posgrados de Calidad (PNPC) del Conacyt:

Sujeto A: Empezamos a hacer todos los trámites para entrar al PNPC y el problema ahí fue mayor, porque para mantenernos en este, lo más problemático y lo más pesado son los fondos, sí, o sea, necesitábamos fondos, necesitábamos laboratorios, no teníamos laboratorios ¿Dónde trabajamos? Íbamos al laboratorio de [tal área], de [tal otra] a que nos dieran un pedacito así para toda la maestría, para todos los alumnos. [...]. Porque echamos a andar la maestría pero no teníamos nada. Entonces necesitábamos financiamiento, [...], [pero] nos dijeron: no hay financiamiento, $[. .$.$] , pues empezamos.$

\section{Entrevistador: ¿Cómo le hicieron?}

Sujeto A: Pues empezamos a participar en convocatorias, a Fomix [Fondo Mixto Conacyt], a Conacyt [...]. De hecho, si tú ves en todas las convocatorias nosotros siempre convocamos a todo, a todas vamos, porque necesitamos fondos para trabajar con los alumnos. Entonces necesitamos muchos, muchos fondos de Conacyt, de Fomix, de todos. Del cuerpo académico empezamos a traer: $¡$ ah, pues nos aprobaron un proyecto! [...]. A cada uno de nosotros nos aprobaron [varios] proyectos, o sea, teníamos [...] proyectos [...], entonces con eso nos pusimos de acuerdo. ¿Qué necesitamos? Pues que equis, ye y zeta, ok; ¡tú compras equis, yo ye, y tú esto! O sea, empezamos a repartirnos y empezamos a equipar el laboratorio y de hecho [con] parte de mi proyecto, y por ejemplo yo puse para un equipo que todavía tenemos y es de los más usados, yo puse la mitad, [tal profesor] puso la otra mitad y se compró ese equipo. [Otro profesor] compró otro equipo y así. ¡Entonces eso es prácticamente nuestro pan de cada día y es a lo que nos dedicamos!, a tratar de conseguir recursos para equipar los laboratorios para tener dinero para los alumnos para poder comprarles los reactivos. Hemos comprado un material de cinco gramos de un reactivo que necesitamos y costó tres mil pesos. [...] Tú puedes ver que actualmente [...] casi siempre van dos o tres propuestas de [tal área]. Es lo que hacemos actualmente. De que: ¡Oye que ahorita se le aprobó un proyecto a [tal profesor]! ¡Se le aprobó un proyecto a [otro]! ¡Se me aprobó un proyecto a mí! ¡Un proyecto a [a otra maestra]! ¡Un proyecto a [otro investigador]! Ok, en tal laboratorio tal cosa, pues tú cómpralo, nos ponemos de acuerdo en lo que se va comprando. Y así sacamos recursos para desarrollar las tesis de nuestros alumnos".

La falta de mayor financiamiento es un problema recurrente que impacta en todo lo que precisa la informante. Por ejemplo, Asomoza [34] aseveró que había una crisis de financiamiento con la que lamentablemente tenían que lidiar los investigadores nacionales. Reconocer esta problemática es a lo que se refiere el sujeto A cuando expresa en su narrativa que: “¡Entonces eso es prácticamente nuestro pan de cada día y es a lo que nos dedicamos!". En este sentido, lo que expuso Asomoza en su momento respecto a la falta de mayor financiamiento sigue permeando en la vida académica. Sobre todo en ciertas áreas del conocimiento porque se requieren de reactivos y equipo de laboratorio para desarrollar investigación. 
Del testimonio de la informante resalta que ella y sus colegas tratan de subsanar la problemática mediante una postura activa. Su narración da cuenta de la manera en que los académicos del IIT buscan concursar por proyectos de investigación para recibir financiamiento. Su testimonio deja ver una competencia por recursos no solo para adquirir el equipo adecuado para el proyecto, sino también para conseguir los reactivos indispensables. Se identifica una lucha constante por la pertenencia sólida a un grupo que da sentido a un tribalismo [30]. Además, comparten valores, reglas y metas comunes en una organización académica [35]. Desde luego, la búsqueda de recurso para investigar y publicar también lleva el sentido de mantenerse o escalar en el SNI, así como sobresalir en la profesión académica. La competencia con otros investigadores o grupos de investigación por proyectos para obtener financiamiento para el desarrollo de investigación científica y tecnológica se hace cada vez más latente.

Por último se percibe en el testimonio del sujeto A una aportación equilibrada de cada uno de los integrantes de su cuerpo académico, es decir, cada uno contribuye de manera equitativa al grupo para perseguir la permanencia en el SNI.

\subsection{Del publish or perish al direct thesis or perish. El estancamiento de la distinción}

Algunos de los informantes precisaron que para subir de nivel en el SNI es indispensable, además de un número consiste de publicaciones de calidad, dirigir tesis fundamentalmente de posgrado. El PTC que tiene la candidatura busca obtener el nivel I, quien lo tiene busca ascender al nivel II y así sucesivamente. Sin embargo, si mantenerse en el SNI es complicado ante las problemáticas aquí documentadas y escalar los distintos niveles es aún más. Por tanto, es fundamental participar en programas de posgrado como tutores de tesis. Una entrevistada (sujeto A) compartió que la ausencia de este aspecto limita sus posibilidades de pasar del nivel I al II:

Pues mira, la vez pasada [hace cuatro años] en mi evaluación no me dieron el nivel II porque no tenía alumnos del doctorado, eso fue lo que me contestaron. [...]. Yo tenía las citas que te piden, yo tenía todas las citas, yo tenía el número de artículos y lo único que me mandaron decir es que me faltaron alumnos de doctorado para poder tener el nivel II. Entonces, desde la vez pasada [...], si desde hace cuatro años $[\ldots]$, por ejemplo, si yo hubiera trabajado en una institución como del centro, ¿no?, que no estuviera la problemática así [...], hubiera tenido alumnos de doctorado [y] yo hubiera sido hace cuatro años nivel II. Sin embargo, es una limitante y por ejemplo esta situación Conacyt no la ve. [...].

El testimonio del sujeto A es revelador. Argumenta una desventaja con investigadores del centro del país en cuanto a la formación de recurso humano que no ponderan las comisiones dictaminadoras del SNI. Solo recomiendan en los dictámenes dirigir más tesis de posgrado para ascender de nivel, pero cada académico y cada institución tienen su propia problemática y su propio contexto. Estas limitaciones desde luego impactan en el resultado de la evaluación en el SNI. Para dirigir múltiples tesis se requieren de condiciones. El sujeto A expuso una dificultad:

... hay poca gente que quiere venir a estudiar maestría y doctorado. Y si no tenemos alumnos de maestría y doctorado no publicamos, si no publicamos no estamos en el SNI I, pero para pasar al nivel II necesitamos alumnos de doctorado, si no tenemos alumnos de doctorado nunca vamos a poder pasar al nivel II. Entonces, los de doctorado, por ejemplo, los que tengo yo de doctorado son alumnos que han hecho la tesis conmigo en la maestría y en la licenciatura. Tengo alumnos de licenciatura y luego que van a la maestría y luego empiezan el doctorado [...], porque son gente que viven ya aquí, no vienen de afuera como en otras partes.

En suma, la formación de recurso humano resulta clave para ascender de nivel en el SNI. Al interpretar el testimonio del sujeto A y otros entrevistados, se desprende que pertenecer al núcleo académico básico (NAB) de un posgrado con determinada antigüedad y cierto grado de consolidación influye favorablemente en las evaluaciones de los PTC por parte del SNI, ya que hay recurso humano para dirigir tesis. De esta forma, ascender de nivel es posible.

En las narrativas de los entrevistados fue posible identificar que una buena parte de los académicos experimentan un estancamiento como investigadores nacionales tras el resultado de los dictámenes. Si bien se mantienen dentro del Sistema y han renovado el nombramiento en varias ocasiones, no es lo que esperan recibir por parte de los evaluadores. Ellos aspiran al siguiente nivel por- 
que afirman tener las suficientes publicaciones y citas reportadas ante el SNI. En cambio, este es determinante al recomendarles que necesitan reunir el requisito de formar recurso humano para conseguirlo.

Finalmente, a partir del análisis de los datos emergentes con base en la grounded theory como metodología, se desprende el concepto de estancamiento de la distinción. Lo defino como la circunstancia experimentada por aquellos académicos, que teniendo la productividad académica suficiente, no han podido pasar del nivel I al nivel II dentro del SNI luego de haber renovado en algunas ocasiones el nombramiento como investigador nacional. Esto por adolecer del requisito de la formación de recurso humano en el nivel de posgrado.

\section{CONCLUSIONES}

En suma, es clara la rigurosidad de los criterios de evaluación de las áreas del conocimiento en las que son evaluados la mayoría de los académicos del IIT que pertenecen al SNI. A partir de los resultados presentados en este artículo, resaltan las problemáticas particulares que viven y experimentan para ingresar, mantenerse dentro del SNI o escalar.

Si bien es cierto que los académicos de otras áreas del conocimiento afrontan problemáticas parecidas y fue discutido en el apartado introductorio donde se presenta una revisión de la literatura publicada, en este trabajo se documentan múltiples aspectos que diferencian las experiencias de los PTC que realizan actividades académicas en alguna rama de la ingeniería en el contexto del IIT con los PTC de otros entornos. Por tanto, el objetivo del artículo se ha cumplido eficazmente.

Sin embargo, una de las limitaciones de este trabajo es que deriva de un proceso de investigación cualitativa de corte interpretativo en relación a una determinada muestra de informantes, por lo que no es posible generalizar los resultados ni las conclusiones. Sin embargo, esto no significa que las problemáticas, experiencias e interpretaciones aquí documentadas no ocurran en otras organizaciones académicas del país. En este sentido, el artículo contribuye a su comprensión, ya que existe una ausencia relativa de trabajos bajo metodologías cualitativas y, sobre todo, utilizando la grounded theory, que analicen las experiencias y problemáticas de los académicos que realizan actividades de investigación en alguna de las ramas de la ingeniería.
Finalmente, a partir de los hallazgos encontrados, se recomienda que - a nivel institucional- se deben emprender políticas y acciones en materia de investigación científica que sean diferenciadas según las necesidades y requerimientos de los institutos, de las áreas disciplinares y de las líneas de generación y aplicación del conocimiento que ejercitan los y las investigadores/as de la universidad. Esto en razón de que investigar en ciertas áreas implica la utilización de reactivos costosos que, sin ellos, el proceso de investigación se detiene o simplemente no se puede llevar a cabo. Además, la política institucional en la materia debe articularse en conjunto con otros aspectos y decisiones institucionales que, aunque parecieran colaterales o adyacentes, desde luego impactan de manera determinante en el desarrollo de investigación, en la generación de productividad y en el logro de indicadores institucionales que toda organización académica busca de manera imprescindible.

\section{REFERENCIAS}

[1] Conacyt, Reglamento del Sistema Nacional de Investigadores, Ciudad de México, 2020. [En línea]. Disponible en: https://www.conacyt.gob.mx/index.php/ el-conacyt/sistema-nacional-de-investigadores/marcolegal/reglamento-sni/20142-reglamento-sni/file

[2] Conacyt, Criterios SNI, Ciudad de México, 2019. [En línea]. Disponible en: https://www.conacyt.gob.mx/index. php/el-conacyt/sistema-nacional-de-investigadores/ marco-legal/criterios-sni

[3] H. Camarillo, “ ¿Por qué ingresar a la élite de investigadores? Seis motivos documentados en una universidad mexicana", Areté, vol. 6, no. 12, pp. 203-225, ago. 2020. Disponible en: http://saber.ucv.ve/ojs/index.php/rev_ arete/article/view/19403

[4] SEGOB, Acuerdo por el que se establece el Sistema Nacional de Investigadores, Diario Oficial de la Federación 26/07/1984, Ciudad de México, 2019. [En línea]. Disponible en: http://dof.gob.mx/nota_detalle. php? codigo $=4680072 \&$ fecha $=26 / 07 / 1984$

[5] M. Gil y L. Contreras, "El Sistema Nacional de Investigadores: ¿espejo y modelo?”, Revista de la Educación Superior, vol. 46, no. 184, pp. 1-19, dic. 2017. Disponible en: http://resu.anuies.mx/ojs/index.php/ resu/article/view/13 
[6] J. Galaz y M. Gil, "La profesión académica en México: un oficio en proceso de reconfiguración”, Revista Electrónica de Investigación Educativa, vol. 11, no. 2, pp. 1-31, jul. 2009. Disponible en: https://redie.uabc.mx/ redie/article/view/237/396

[7] S. Didou y E. Gérard, El Sistema Nacional de Investigadores, veinticinco años después. La comunidad científica, entre distinción e internacionalización. México: ANUIES, 2010.

[8] M. De Ibarrola, "La evaluación del trabajo académico desde la perspectiva del desarrollo sui géneris de la educación superior en México", Perfiles Educativos, no. 5354, pp. 1-6, jul.-dic. 1991. Disponible en: https://www. iisue.unam.mx/perfiles/numeros/1991/53-54

[9] H. Camarillo, "Permanecer en el Sistema Nacional de Investigadores. Diferencias en los entornos institucionales", Cuadernos Fronterizos, vol. 9, no. 27, pp. 36-39, 2013. Disponible en: https://erevistas.uacj.mx/ojs/index.php/cuadfront/article/view/1890

[10] M. Gil, "Diferenciar para Reconocer", Perfiles Educativos, no. 53-54,pp. 35-39, jul.-dic. 1991. Disponible en: https:// www.iisue.unam.mx/perfiles/numeros/1991/53-54

[11] R. Glazman, "Evaluación académica, estímulos y becas: los programas de pago por rendimiento en las universidades", Perfiles Educativos, no. 53-54, pp. 58-61, 1991. Disponible en: https://www.iisue.unam.mx/perfiles/numeros/1991/53-54

[12] Á. Díaz Barriga, "Los programas de evaluación (estímulos al rendimiento académico) en la comunidad de investigadores. Un estudio en la UNAM", Revista Mexicana de Investigación Educativa, vol. 1, no. 2, pp. 408-423, jul.-dic. 1996. Disponible en: http://www.comie.org. $\mathrm{mx} / \mathrm{v1} / \mathrm{revista} /$ visualizador.php? articulo=ART00201\&criterio=http://www.comie.org.mx/documentos/rmie/v01/n002/pdf/rmiev01n02scC00n08es.pdf

[13] W. De Vries, "Silencios y Ruidos: Las Políticas para la Educación Superior en México", Revista de la Educación Superior, no. 114, pp. 1-11, jun. 2000. Disponible en: http://publicaciones.anuies.mx/revista/114/2/3/es/silencios-y-ruidos-las-politicas-para-la-educacion-superior-en-mexico
[14] R. Grediaga, "Retos y condiciones de desarrollo: la profesión académica en México en la última década”, Revista Mexicana de Investigación Educativa, vol. 6, no. 11, pp. 95-117, ene.-abr. 2001. Disponible en: https:// www.redalyc.org/articulo.oa?id=14001107

[15] S. Salord, "Las trayectorias académicas: de la diversidad a la heterogeneidad", Revista Mexicana de Investigación Educativa, vol. 6, no. 11, pp. 1-13, ene.-abr. 2001. Disponible en: https://www.redalyc.org/articulo. oa? id=14001103

[16] D. E. Magaña y P. A. Sánchez, "Síndrome de desgaste emocional en investigadores mexicanos", Revista Interamericana de Psicología, vol. 42, no. 2, pp. 353362, 2008. Disponible en: https://www.redalyc.org/ pdf/284/28442216.pdf

[17] H. Camarillo y R. Rincones, "Ingresar al Sistema Nacional de Investigadores. Voces de los académicos de la UACJ", en Chihuahua Hoy. Visiones de su historia, economía, política y cultura, V. Orozco, coord. México: UACJ/UACH/Instituto Chihuahuense de la Cultura, 2014, pp. 477-514.

[18] V. Cárdenas, "Motivaciones para ingresar al SNI. Un estudio de caso con investigadores jóvenes", Sinéctica. Revista Electrónica de Educación, no. 44, pp. 1-17, jul. 2015. Disponible en: https://sinectica.iteso.mx/index. php/SINECTICA/article/view/155

[19] H. Camarillo, "Implicaciones de la evaluación académica. Percepciones y preocupaciones de los profesores de tiempo completo de la Universidad Autónoma de Ciudad Juárez acerca de su permanencia en el SNI”, Sinéctica. Revista Electrónica de Educación, no. 44, pp. 1-21, jul. 2015. Disponible en: https://sinectica.iteso. mx/index.php/SINECTICA/article/view/156

[20] J. Rodríguez, C. González y G. Maqueda, "El Sistema Nacional de Investigadores en México: 20 años de producción científica en las instituciones de educación superior (1991-2011)", Investigación Bibliotecológica, no. esp., pp. 187-219, 2017. DOI: 10.22201/iibi.24488321xe.2017.nesp1.57890

[21] I. Izquierdo y M. Atristan, "Experiencias de investigadoras en su ingreso, promoción y permanencia en el 
Sistema Nacional de Investigadores: tensiones y estrategias identitarias", IE. Revista de Investigación Educativa de la REDIECH, vol. 10, no. 18, abr.-sep. 2019. DOI: 10.33010/ie_rie_rediech.v10i18.466

[22] A. Strauss y J. Corbin, Bases de la investigación cualitativa. Técnicas y procedimientos para desarrollar la teoría fundamentada, 1. ${ }^{\mathrm{a}}$ ed. en español. Antioquía: Editorial Universidad de Antioquía, 2002.

[23] UACJ, Instituto de Ingeniería y Tecnología, Universidad Autónoma de Ciudad Juárez, Ciudad Juárez, Chih., 2020. [En línea]. Disponible en: http://www3.uacj.mx/ IIT/Paginas/default.aspx

[24] Conacyt, Archivo histórico del Sistema Nacional de Investigadores (SNI), Ciudad de México, 2019. Consultada en: 2020. [En línea]. Disponible en: https:// www.conacyt.gob.mx/index.php/el-conacyt/sistemanacional-de-investigadores/archivo-historico

[25] E. Garfield, "What Is The Primordial Reference For The Phrase 'Publish Or Perish'?”, The Scientist, vol. 10, no. 12, jun. 1996. Disponible en: www.garfield.library.upenn. edu/commentaries/tsv10\%2812\%29p11y19960610.pdf

[26] E. L. Boyer, Una propuesta para la educación superior del futuro. México: Fondo de Cultura Económica, 1997.

[27] G. Reyes y J. Suriñachi, "Las publicaciones de los investigadores mexicanos en el ISI: realidad o mito del SNI". Revista Electrónica Sinéctica, no. 38, pp. 1-30, ene.-jun. 2012. Disponible en: https://www.redalyc.org/ pdf/998/99824765005.pdf

[28] R. Miranda, La frustración y la envidia. Política, decisiones y estímulos a la actividad académica. México: Universidad de Guadalajara, 2005.
[29] R. Day, Cómo escribir y publicar trabajos científicos. Washington, D. C: Organización Panamericana de la Salud, 2005.

[30] M. Maffesoli, El tiempo de las tribus. El ocaso del individualismo en las sociedades posmodernas. México: Siglo XXI, 2004.

[31] E. Luna-Morales, J. M. Russell y C. Mireles-Cárdenas, "Evolución e impacto de la investigación en la Universidad Autónoma de San Luis Potosí, México. Patrones de publicación y Sistema Nacional de Investigadores", Investigación Bibliotecológica, vol. 26, no. 58 , pp. $175-213$, sep.-dic. 2012. Disponible en: DOI: 10.22201/iibi.0187358xp.2012.58.35985

[32] B. Musi-Lechuga, J. A. Olivas-Ávila, G. Ochoa-Meza, R. Espinoza-Ornelas y B. Caraveo-Camarena, "Producción científica en psicología de los miembros del Sistema Nacional de Investigadores de México", Revista Latinoamericana de Psicología, vol. 50, no. 2, pp. 117125, 2018. DOI: 10.14349/rlp.2018.v50.n2.5

[33] E. Cabrero, "Principales logros y desafíos del Sistema Nacional de Investigadores de México a 30 años de su creación", Revista Iberoamericana de Ciencia, Tecnología y Sociedad, vol. 10, no. 28, ene. 2015. Disponible en: https://www.redalyc.org/pdf/924/92433772013.pdf

[34] R. Asomoza, "20 años del Sistema Nacional de Investigadores. Impacto y perspectiva", en Una reflexión sobre el Sistema Nacional de Investigadores a 20 años de su creación, 1. a ed. México: FCCT y AMC, 2005, pp. 133-135. Disponible en: http://www.coniunctus.amc. edu.mx/libros/20_sni_final.pdf

[35] J. March y J. Olsen, El redescubrimiento de las instituciones. La base organizativa de la política. México: FCE, 1997. 